\title{
Security EarlyWarning Assessment Progrm of Hazardous Sources Based on EarlyWarning Information Processing Model
}

\author{
Jia Ling \\ The Armed Police Academy \\ Langfang, China \\ jialing123321@163.com
}

\begin{abstract}
The early warning can constantly improve the monitoring and management of the hazardous sources to realize advance prevention and control. Many early warning methods do not predict and imitate the hazard sequel and the early warning information in incomplete. This essay analyzes and studies the early warning process of the hazardous sources, hazard assessment techniques and analyzes the hazardous sources quantitatively and qualitatively. This essay combines effectively the imitation of the hazard sequel and the early warning technology, proposes hazardous sources security early warning procedure information processing system structure and designs models for processing early warning information of the hazardous sources, improving the early warning of the hazardous sources and increasing the accuracy and efficiency of the prior warning. Combining with cases, it performs imitation of the hazard sequel of leakage accident. Practices have shown that the proposed imitated early warning structure and methods are efficient. The imitation result is nearly matched with the fact. The early warning gets satisfied effect having certain practical value.
\end{abstract}

Keywords-security prior warning; early warninginformation; assessment program; model

\section{INTRODUCTION}

The early warning theory is as a result of the war. After the World War II, America firstly applied the early warning theory in the economy field which provided effective macro economy operation decision support. Later, Britain, EC and other countries gradually applied it to many fields and yielded good effect. Security management system of the hazardous sources is a complex system, which involves with many aspects such as the environment, devices, staffs and management and so on. The relevant studies have been started from the identification ${ }^{[1,2]}$ of the hazard sources, classification $^{[3]}$, assessment ${ }^{[4]}$, monitoring and prior warning ${ }^{[5]}$ and so on domestically and internationally. Study on some steps and profession has been mainly focused on respectively. Many practical achievements have been obtained.

With the actual demand of the security management of the hazardous sources, the early warning information is incomplete that early warning is performed by informing the hazard in advance after only finding out or calculating the critical threshold of the hazard. The taken early warning measurements are with weak points, especially, there are no corresponding danger strategies for the caused hazard sequel. The effective early warning strategies and measurements can not be formed. If the dangerous sequel caused by the hazardous sources can be calculated or imitated, more complete and accurate data will be provided for decision and reference. Adopting prediction calculation method ${ }^{[6]}$ to get relevant data and combining the imitation of the hazard sequel with the early warning of the hazard sequel, except the accident ratio can be decreased, the severity of the hazard sequel can also be reduced, raising the management level of the hazardous sources. Therefore, an imitated early warning method is proposed to try to perform early warning of the hazardous sources.

\section{STRUCTURE OF SECURITY EARLY WARNINGSYSTEM OF HAZARDOUS SOURCES}

According to reference [5], early warning process mainly includes the definiteness of the warning, looking for the warning sources, the analysis of the warning and the forecast of the warning degree. References [7] and [8] have designed an imitated early warning system, as shown in figure 1 . The system includes data acquisition of the hazard information, hazard identification, hazard consequence imitation, comprehensive analysis and hazard assessment, early warning and control of the hazard based on the strategies. Combining the consequence imitation with the early warning to make the early warning information more complete, detailed, make the warning with stronger points and provide conveniences for making scientific early warning measurements.

\section{A. Data Aquisition}

Data acquisition is to collect automatically the information of the environment or safety state of the hazard sources mainly through the special devices or monitoring system. The collected data is usually arranged to be used by other relevant processes in the early warning process.

\section{B. Hazard Identification}

The hazard identification ${ }^{[9]}$ means that identifying the major hazard sources based on the data acquisition and judging and recognizing the hazard through qualitative analysis and combined with experiences.

(1) identifying major hazard sources of chemicals category

Reference to "Major Accident Hazard Act in Industry Activities" in EC in 1982, "State Standard for Controlling Major Hazard Sources" in Australia in 1996 and GB 182182000 "Major Hazard Source Identification" in our country, hazard identification is mainly by whether the number of the 
calculated hazardous substance within unit is over the critical quantity.

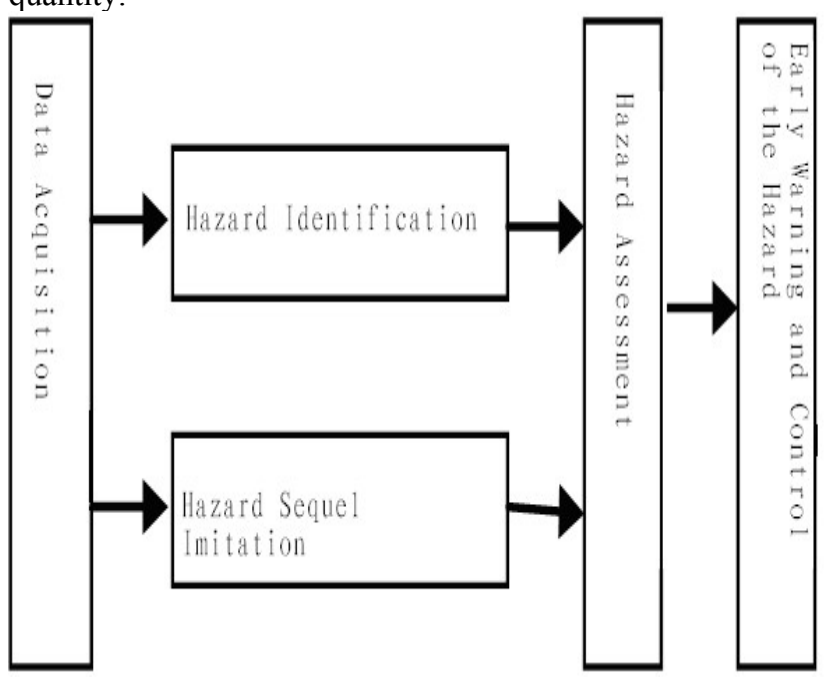

Figure 1. Imitated prior warning structure

Identification process is as follows:

(1) for $n$ kinds of chemicals, $i=1,2, \ldots n, q_{i}$ is the practical storage of the chemicals, $Q_{i}$ is the critical quantity of hazard of the storage of the chemicals corresponding to $\mathrm{q}_{\mathrm{i}}$;

(2) calculate $\mathrm{t}=\mathrm{q}_{\mathrm{i}} / \mathrm{Q}_{\mathrm{i}}, \quad \forall \mathrm{t}$, if $\exists \mathrm{t} \geq 1$, belonging to hazardous chemicals;

(3) for $\mathrm{t}=\mathrm{q}_{\mathrm{i}} / \mathrm{Q}_{\mathrm{i}}, \forall \mathrm{t}$, if $\exists \mathrm{t}<1$, calculate $\mathrm{k}=\sum_{i=1}^{n} q_{i} / Q_{i}$, and $\mathrm{k} \geq 1$, belonging to hazardous chemicals;

(4) otherwise, not belonging to hazardous chemicals.

(2) identifying major hazard sources of non-chemicals categories

At present, domestically, the identification of the nonchemicals categories such as hazard buildings, pressure pipeline and containers, ray source, dangerous places and so on involves many factors. Except some self-owned important technical parameters, other factors such as environment factor, how the factor affects another, operation of the staffs, management quality, ability and level and so on shall be included. No basis for identification has specifically been proposed. But comprehensive assessment can be performed by adopting fuzzy theory and level analysis and then judge.

\section{Security Assessment of Hazard Sources}

Up to dozens of security analysis and assessment methods have been put forward domestically and internationally, such as security distance assessment method, assessment method based on accident sequel, assessment method based on risks, assessment method of inflammable, explosive, toxic and other major hazards. In analysis and assessment, qualitative and quantitative analysis or the analysis of combining the two are mainly adopted. The qualitative analysis includes Preliminary Hazard Analysis (PHA), Safety Checklist (SCL), Operation Hazard Analysis (OHA), and Fault Hazard Analysis (FHA). The quantitative analysis includes Fire and Explosion Index Method used in
DOW Chemical Industry Company in America, ICI Mond Method used in Imperial Chemical Company in Britain, SixStage Security Assessment Method used in the Labor Ministry of Japan. The analysis that combines the qualitative analysis with the quantitative analysis includes Fault Tree Analysis method (FTA) and Event Tree Analysis method (ETA).

The quantitative analysis is to calculate the probability of the fault and event to get the risk degree. The use of the order of magnitude to express the security can get relative high accuracy. But because there is lack of necessary event data at present, there is a certain difficulty for applying it in practice. The qualitative analysis is mainly performed according to work experiences and personal judgment, simple, easy to operate, intuitive assessment results, but great randomness and affected by subjective factors easily. It can be seen that the existing methods are just used for dangerous or hazardous unit or system, by which, the hazard degree is expressed by hazard grade and the hazard sequel can not be described quantitatively and intuitively. It is more effective to improve security early warning management by using reasonable mathematical model to imitate the hazard, by using the corresponding injure model to assess the effects of the caused hazard, and by using figures and tables and other intuitive methods to describe the influenced area, casualties and property losses and so on.

\section{Hazard Sequel Imitation}

The hazard sequel imitation is based on the data acquisition, using related hazard sources, hidden danger or accident simulation mathematical model and calculation techniques to imitate the process and calculate the relevant parameters of the hazard sequel to predict the relevant situations of caused hazard, such as hazard area, leakage rate, death radius and property loss and so on.

\section{E. Hazard Early warningand Control}

Based on the collected data, identify the data of the results and hazard imitation sequel to comprehensively analyze according to the facts to make feasible and effective hazard control strategies timely to guide and conduct the hazard sources management work. Through technology measurements, security management, emergency preplan with strong points, the hazard sources accidents can be predicted and emergency methods can be applied to process the post events. Ensure the implementation effect of the security strategies to improve the overall hazard sources management level to realize efficient security hazard sources management level at last.

\section{EARLY WARNINGINFORMATION PROCESSING MODEL}

Hazard sources early warning is realized by that on the basis of inspecting the relevant state information of the hazard sources in real time, the mathematical model describing the security state and its simulation program show intuitively whether the hazards convert towards the critical state of the accidents according to the variation tendency of the state information and give out 
corresponding early warning information or emergency control instruction timely.

Early warning mainly includes risk information acquisition, hazard identification, hazard sequel imitation, security assessment, strategy matching. There is dependency between these processes. Therefore, define the early warning information processing model EWM (Early Warning Model of Dependency) based on dependency.

Definition 1: EWM=Relation (Input, Output), in which, Output $=$ (InputParameter, HazardSequel, EvaluateGrade, SafetyControl)

Relation is dependency relationship, referring to some relation, such as hazard identification, sequel imitation, hazard assessment, security strategy matching; Input and Output are the leading-in of the input parameters and the output of the results; InputParameter $=\left\{\mathrm{v}_{1}, \mathrm{v}_{2}, \ldots, \mathrm{v}_{\mathrm{n}}\right\}$ is the collected hazards parameter V. HazardSequel $=\{\mathrm{h} 1, \mathrm{~h} 2, \ldots, \mathrm{hm}\}$ is the sequel imitation performed based on the collected InputParameter parameter to form hazard result data $\mathrm{H}$, as the important early warning parameter and a basis for making safety strategy and measurements. EvaluateGrade $=\{\mathrm{d} 1, \mathrm{~d} 2, \ldots, \mathrm{dj}\}$, generally, $\mathrm{j}$ refers to the grade. Hazard grade $\mathrm{D}$ is the evaluation based on the collected parameter $\mathrm{V}$ and the hazard sequel imitation result $\mathrm{H}$, as an essential basis for management and early warning. The security strategy base $\mathrm{P}$ of the hazardous sources management formed based on state standard, industry requirement and experiences matches the corresponding strategy through the sizes of the weights according to $h_{i}$. Therefore, safety strategy B with strong points is formed based on P. SafetyControl $=$ SafetyControl $=\{b 1, b 2, \ldots, b m\}$, implement prior prevention or early warning and emergency process after accidents.

$\mathrm{F} 1: \mathrm{V} \rightarrow \mathrm{H}, \quad(\mathrm{h} 1, \mathrm{~h} 2, \ldots, \mathrm{hk})=\mathrm{fl}(\mathrm{v} 1, \mathrm{v} 2, \ldots, \mathrm{vn}) ; \mathrm{f} 1()$ is the mathematical expression of sequel imitation of the hazardous sources.

F2:

$\mathrm{D},(\mathrm{d} 1, \mathrm{~d} 2, \ldots, \mathrm{dm})=\mathrm{f} 2(\mathrm{v} 1, \mathrm{v} 2, \ldots, \mathrm{vn}, \mathrm{h} 1, \mathrm{~h} 2, \ldots, \mathrm{hk}) ; \mathrm{f} 2()$ is the mathematical expression of safety grade of the hazardous sources.

F3:

$(\mathrm{V}, \mathrm{H}, \mathrm{D}, \mathrm{P})$

$\mathrm{B}$,

$(\mathrm{b} 1, \mathrm{~b} 2, \ldots, \mathrm{bm})=\mathrm{f} 2(\mathrm{v} 1, \mathrm{v} 2, \ldots, \mathrm{vn}, \mathrm{h} 1, \mathrm{~h} 2, \ldots, \mathrm{hk}, \mathrm{d} 1, \mathrm{~d} 2, \ldots, \mathrm{dm}, \mathrm{p} 1$, $\mathrm{p} 2, \ldots, \mathrm{pm}) ; \mathrm{f} 3()$ is the mathematical expression of the formation of the safety strategy of the hazardous source.

\section{HAZARD SEQUEL IMITATION}

Some area is located at $29.37^{\circ} \mathrm{N}$ and $113.1^{\circ} \mathrm{E}$ with average temperature $17^{\circ} \mathrm{C}$ and average yearly wind speed $3 \mathrm{~m} / \mathrm{s}$. There are 50 tons of liquid ammonia in the ammonia tank with an irregular triangle with a crack of $3.3 \mathrm{~cm}$ length and $1.2 \mathrm{~cm}$ width. The leakage occurred at $16: 00 \mathrm{pm}$ of June $7^{\text {th }}$. It was cloudless and clear with a southwestern wind.

\section{A. Hazard Identification}

According to the state standard, the critical quantity of the ammonia (storage tank) is $20 \mathrm{~T},(50 / 20)>1$, belonging to hazard substances.

\section{B. Hazard Imitation}

\section{a) Calculation of Leakage Quantity}

The maximum charge pressure of the liquid ammonia is not over 1.6 Mpa. According to the sonic flow formula (1) of the gas leakage, the gas leakage rate shall be calculated.

$$
Q=C_{d} A P \sqrt{\frac{M k}{R T}\left(\frac{2}{k+1}\right)^{\frac{k+1}{k-1}}}
$$

In which, $\mathrm{Q}$ is the gas leakage rate $(\mathrm{kg} / \mathrm{s}), \mathrm{Cd}$ is the gas leakage density, $\mathrm{A}$ is the crack area $\left(\mathrm{m}^{2}\right), \mathrm{M}$ is the relative molecular mass of the gas, $\mathrm{R}$ is universal gas constant $\left(8.31436 \mathrm{Jmol}^{-1} \mathrm{~K}^{-1}\right), \mathrm{T}$ is the storage temperature $(\mathrm{K})$ of the gas.

$\mathrm{A}=0.5 \times 3 \times 10-2 \times 1 \times 10-2=1.5 \times 10-4\left(\mathrm{~m}^{2}\right) ; \mathrm{P}=1.6 \times$ 106 ; $\mathrm{Cd}=0.95$ (the crack is a triangle in shape); $\mathrm{K}=1.839$ ( according to checking the adiabatic index and the critical pressure); $\mathrm{M}=0.017 ; \mathrm{T}=273+16.5=289.5$.

According to formula (2), the leakage rate: $\mathrm{Q}=0.7483 \mathrm{~kg} / \mathrm{s}$

Supposed if the pressure of the container does not change, the time for the leakage of the 50 tons of the liquid ammonia shall be: $50 * 1000 / 0.344 \approx 18.56$ hour.

If the leakage lasts for 30 minutes, the total leakage quantity shall be up to $0.7483 \times 1800=1197.3 \mathrm{~kg}$

The leakage will cause how large area to be hazardous, the following imitation of the diffusion of the leakage will be adopted to tell.

b) Imitated Sequel of the Diffusion of the Hazard

s. Check the incidence meter of the sun and get the incidence of the sun $\delta=22$. The solar altitude shall be calculated by formula (3):

$h=\arcsin [(\sin \phi \cdot \sin \delta+\cos \phi \cdot \cos \delta \cdot \cos (15 t+\lambda-300)]$

In which, $\varphi$ and $\lambda$ refer to longitude and latitude respectively and $\mathrm{t}$ is the observation time

The solar altitude $\mathrm{h}$ shall equal to 47.6. The gas tank is on the ground. If leakage occurs, it equals to the diffusion model of the continuous point source on the ground. The mathematical model is shown as formula (4)

$$
C(x, y, z)=\frac{Q}{\pi V \delta_{y} \delta_{z}} \cdot \exp \left[-\left(\frac{y^{2}}{2 \delta_{y}^{2}}+\frac{z^{2}}{2 \delta_{z}^{2}}\right)\right]
$$

In which, $\delta_{y}$ and $\delta_{z}$ refer to the diffusion coefficients of the pollutants spread towards directions $\mathrm{y}$ and $\mathrm{z}$ respectively. $\mathrm{m}$, $\mathrm{Q}$ refer to the source strengths, $\mathrm{mg} / \mathrm{s}$. V refers to the mean wind speed, $\mathrm{m} / \mathrm{s}$.

According to the atmospheric stabilities grades, the expressions of the diffusion coefficients $\delta_{y}$ and $\delta_{z}$ can be 
inquired. According to GB Z2-2002 Hazard Occupational Exposure Limit in Working Sites, the maximum concentration of the ammonia shall be $30 \mathrm{mg} / \mathrm{m}^{3}$; when in $500 \mathrm{mg} / \mathrm{m}^{3}$, people will die from breathing in it within 5 minutes. Therefore, $30 \mathrm{mg} / \mathrm{m}^{3}$ is as the diffusion concentration limit value and $500 \mathrm{mg} / \mathrm{m}^{3}$ is the death area. Therefore, the toxic areas and the death distance of $0-500 \mathrm{~m}$, $500-1000$, more than $1000 \mathrm{~m}$ from the leeward distance can be calculated.

For example, within $0-500 \mathrm{~m}$, leakage for one second, the toxic area and the death distance can be calculated as follows:

$$
\begin{aligned}
& 30=\frac{4.54 \times 10^{5}}{3.14 \times 3 \times 0.2295 x^{0.919325} \times 0.114682 x^{0.941015}} \times \\
& \exp \left[-\left(\frac{y^{2}}{2 \times\left(0.2295 x^{0.919325}\right)^{2}}+\frac{z^{2}}{2 \times\left(0.114682 x^{0.919325}\right)^{2}}\right)\right]
\end{aligned}
$$

Let $\mathrm{z}=0$, the mathematical expression of the toxic areas can be obtained:

$$
y^{2}=1.16114 x^{1.83865}-0.196032 x^{1.83865} \ln x
$$

Similarly, the mathematical expression of the death area can be obtained:

$$
y^{2}=0.86462 x^{1.83865}-0.196032 x^{1.83865} \ln x
$$

From the calculation of the imitation results by adopting MATLAB software, diffusion to $360-370 \mathrm{~m}$ leeward belongs to toxic areas; diffusion to within $80 \mathrm{~m}$ leeward belongs to death areas.

Compared with the historical data, the imitation results is basically close to the facts. Actually, the wind speed is specially important. The faster the wind speed is, the faster it spread. Then the toxic and death area will narrow.

\section{Loss Estimation}

Divide the areas around the hazardous sources into uniformly-spaced net regions. The casualties can be calculated as formula (7):

$$
N=\sum_{i=1}^{n} D_{i} \cdot S \cdot v_{i}
$$

In which, $\mathrm{N}$ refers to the total number of the deaths, $\mathrm{D}_{\mathrm{i}}$ is the population density in the $i^{\text {th }}$ net region, $S$ is the area of the net, $\mathrm{V}_{\mathrm{i}}$ is the personal death rate, $\mathrm{n}$ is the number of the nets.

Calculate $\mathrm{N}$, according to the national grades division methods of the hazard sources, the safety grade of the hazard sources can be divided. When $\mathrm{N}$ equals to 8 (here the calculation process is ignored), the safety grades are divided into as the three grade.

\section{Early Warning and Control of the Hazard}

According to the analysis above, through matching early warning strategy, the relevant hazard control measurement can be got.
1) safety distance. Pay attention to the topography, nature environment, especially the yearly wind directions when storing. Make sure certain safety distance in the leeward direction.

2) crash time. Master the time of the occurring and diffusion of the leakage and specify the corresponding crash time to deal with the emergency.

3) casualties. From the diffusion ranges, combining the population density, the number of the casualties and related property loss can be estimated.

\section{CONCLUSION}

Through analyzing and studying the early warning process of the hazard sources and the early warning techniques, combining with the information processing technology, combining the sequel imitation and the early warning, this essay proposes a kind of imitated early warning system structure. On the basis of this, the essay designs an early warning information processing model and use MATLAB software to perform computer simulation study and the sequel imitation of the hazardous sources. The practical application has shown that the proposed early warning process information processing structure and information processing model is efficient. They can be used to perform early warning. The imitation calculated result is nearly matched with the fact. The early warning gets satisfied effect, having certain practical value.

\section{REFERENCES}

[1] GB18218-2000, "Indentification of Major Hazard Sources" [S]. Beijing: Beijing Plan Press, 2000.

[2] Shi Lichen, Wei Lijun, Luo Aimin. "Discuss about the Problems in the Identification of the Major Hazard Sources" [J]. Journal of Safety and Environment. 2008,2 (8):163-166.

[3] Li Deshun, Xu Kaili. "Study on Grading Technology of Major Hazard Sources" [J]. Risk Management. 2007, 9 (10): 44-47.

[4] Hu Haijun, Cheng Guangxu. "A Comprehensive Model of Safety Assemssment of Hazardous Chemical Sources Based on Level Analysis Method"[J]. Journal of Safety and Environment. 2007, 3(7): 141-144

[5] Tan Jialei. "Assessment of Mine Ventilation System and Study on Security Early Warning System" [D]. Qingdao: Shandong Technology University, 2005

[6] Wanghong, Liu Jinlan, Cao Weidong, Xun Xiuxia. "Early Warning Management Model and Analysis of Airline Flight Delay"[J]. Computer Simulation, 2009.26(4):292-296.

[7] KAFTKA Peter. Risk Management-Indispensable Endeavour to Build and to Ensure Safe Operation of Installations(A).In:WANG Yajun, HUANG Ping, LI Shengcai. Progress in safety science and technology (Vol.IV)[C].Beijing: Seienee Press 2004.2517-2526

[8] Deng Qigen. "Risk Monitoring and Early Warning Technology of Safety Management in Chemical Enterprises" [D] Qingdao: Shandong Technology University. 2006

[9] Wu Zongzhi, Gao Jindong. "Identification and Control of Major Hazard Sources" [M]. Beijing: Metallurgical Industry Press,2001

[10] Lv Kang. "Study on Assessment and Grading Methods of Environment Risks of Major Hazard Sources"[D]. Dalian: Dalian University of Technology. 